\title{
Review: some herbal medicines and plant extracts reduced pain and disability and improved function in osteoarthritis
}

Long L, Soeken K, Ernst E. Herbal medicines for the treatment of osteoarthritis: a systematic review. Rheumatology 2001 Jul;40:779-93.

\author{
QUESTION: In patients with osteoarthritis, are herbal medicines and plant extracts \\ effective and safe?
}

\section{Data sources}

Studies were identified by searching Medline, EMBASE/ Excerpta Medica, Biosis, CINAHL, and the Cochrane Library (all from inception to May 2000); scanning the bibliographies of relevant studies and reviews; and by contacting experts and manufacturers in the field.

\section{Study selection}

Studies were selected if they were randomised controlled trials comparing 1 herbal treatment with a placebo or another active drug in patients with osteoarthritis. Studies focusing on back pain, osteoarthritic conditions of the spine, or on parenterally applied herbal preparations were excluded, as were studies that lacked essential methodological detail such as dosage, baseline data, and clinical end points.

\section{Data extraction}

Data were extracted on patient characteristics, intervention type and duration, and outcomes.

\section{Main results}

12 trials and 2 systematic reviews met the selection criteria. 1 crossover trial comparing Articulin- $F$ (an Ayurvedic herbomineral formulation) with placebo found that active treatment reduced severity of pain $(\mathrm{p}<0.001)$ and disability score $(\mathrm{p}<0.05) .2$ trials compared avocado/soybean unsaponifiables with placebo and found that active treatment led to reduced non-steroidal anti-inflammatory drug consumption and improvement in the functional index $(\mathrm{p}<0.01,1$ trial $)$ and pain (1 trial). A meta-analysis combining data from 3 trials found that capsaicin cream was better than placebo in reducing pain (odds ratio 4.36, 95\% CI 2.77 to 6.88 \{number needed to treat 4 , CI 3 to 5$\}^{*}$ ). 1 other trial comparing capsaicin cream with placebo found that active treatment reduced pain severity $(\mathrm{p}=0.02)$ and tenderness on passive range of motion $(\mathrm{p}=0.03)$ and physician palpation $(\mathrm{p}=0.01)$. 2 trials compared Devil's claw with placebo and found a decrease in pain severity (2 trials) and an increase in mobility (1 trial) with active treatment. 1 trial compared Eazmov with diclofenac and found that Eazmov was inferior to diclofenac for both pain severity $(\mathrm{p}<0.001)$ and disability score $(\mathrm{p}<0.05) .1$ crossover trial compared ginger extract with ibuprofen or placebo and found that ibuprofen was more effective than both ginger extract and placebo. 1 trial compared gitadyl with ibuprofen and found no difference between the treatment groups. 1 systematic review of 6 trials evaluating the effectiveness of phytodolor found that this herbal formulation led to reduced pain, increased mobility, and reduced anti-inflammatory drug consumption. 1 trial compared Reumalex with placebo and found that active treatment led to a reduction in pain $(\mathrm{p}<0.05)$ compared with placebo. 1 trial compared nettle sting with placebo and found that placebo led to greater reductions in both pain $(\mathrm{p}=0.03)$ and disability $(\mathrm{p}=0.003)$. Willow bark ( 1 trial) also led to a reduction in pain compared with placebo $(\mathrm{p}=0.047)$. The incidence of adverse effects for these herbal medicines was low.

\section{Conclusion}

In patients with osteoarthritis, some herbal medicines and plant extracts reduced pain and disability and improved mobility, with a low incidence of adverse effects.

*Numbers calculated from data in article.
Source of funding: no external funding.

For correspondence: Dr L Long, University of Exeter, Exeter, $U K$. L.Long@exter.ac.uk

\section{COMMENTARY}

The review by Long et al provides much needed information on the effects of 11 herbal treatments for osteoarthritis. The only other published review on the effectiveness of these treatments focused on 4 herbal interventions. ${ }^{1}$ Manuals on the use of herbal and other complementary or alternative treatments are now available to health professionals. ${ }^{23}$ Although these publications offer useful guidelines regarding the customary use and therapeutic management of these treatments, only 1 makes reference to any scientific studies of their effectiveness.

Using summary tables, Long et al provide detailed information on the studies reviewed. This assists readers to critique the reported findings and draw their own conclusions. These conclusions might differ in degree from those of the reviewers, who acknowledge the lack of replication for most of the studies but then do not sufficiently emphasise the tentative nature of the results.

On the basis of the findings of Long $e t a l$ and Little $e t a l,{ }^{1}$ nurses and other health workers cannot promote the use of herbal treatments for osteoarthritis. Even in the case of avocado-soybean, capsaicin cream, and phytodolor, the evidence of therapeutic benefit is still limited. However, health professionals are now able to accurately describe the evidence base for clients who enquire, and can give assurance that the scientific community is now taking the evaluation of herbal medicines seriously. The number of clinical trials is increasing and will gradually provide the evidence that the public seeks on the effectiveness of the many herbal preparations that are being popularised for the treatment of this pervasive disease. Nurse investigators are challenged to participate in these research efforts.

Violeta Ribeiro, RN, DNSc Associate Professor, School of Nursing Memorial University of Newfoundland St John's, Newfoundland, Canada

1 Little C, Parsons T, Logan S. Herbal therapy for treating osteoarthritis. Cochrane Database Syst Rev 2001(3):CD002947.

2 Kuhn MA, Winston DE. Herbal therapy and supplements: a scientific $\mathcal{E}$ traditional approach. New York: Lippincott, 2000.

3 Springhouse Corporation. Nursing herbal medicine handbook. Springhouse, Pennsylvania: Springhouse Corporation, 2001. 